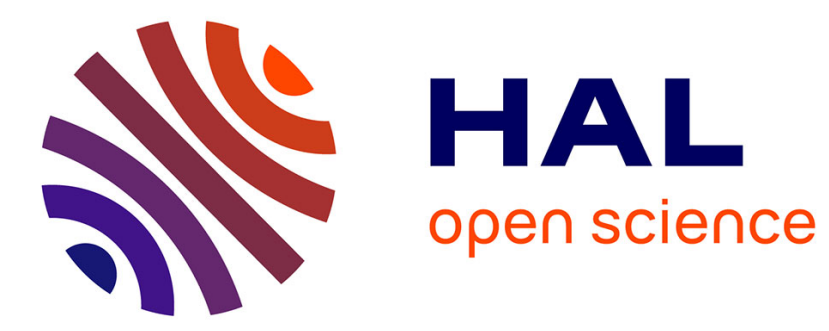

\title{
Étude de l'amorçage des étincelles sous vide à l'aide d'une étincelle auxiliaire produite par une impulsion de courant rectangulaire
}

Jean-Marc Esteva, Michel Bon, Jean-Louis Schwob, Jacques Romand

\section{- To cite this version:}

Jean-Marc Esteva, Michel Bon, Jean-Louis Schwob, Jacques Romand. Étude de l'amorçage des étincelles sous vide à l'aide d'une étincelle auxiliaire produite par une impulsion de courant rectangulaire. Revue de Physique Appliquée, 1970, 5 (2), pp.249-254. 10.1051/rphysap:0197000502024900 . jpa00243383

\section{HAL Id: jpa-00243383 \\ https://hal.science/jpa-00243383}

Submitted on 1 Jan 1970

HAL is a multi-disciplinary open access archive for the deposit and dissemination of scientific research documents, whether they are published or not. The documents may come from teaching and research institutions in France or abroad, or from public or private research centers.
L'archive ouverte pluridisciplinaire HAL, est destinée au dépôt et à la diffusion de documents scientifiques de niveau recherche, publiés ou non, émanant des établissements d'enseignement et de recherche français ou étrangers, des laboratoires publics ou privés. 


\title{
ÉTUDE DE L'AMORÇAGE DES ÉTINCELLES SOUS VIDE A L'AIDE D'UNE ÉTINCELLE AUXILIAIRE PRODUITE PAR UNE IMPULSION DE COURANT RECTANGULAIRE
}

\author{
par M. Jean-Marc ESTEVA, M. Michel BON, \\ M. Jean-Louis SCHWOB et M. Jacques ROMAND \\ Laboratoire des Hautes Pressions, C. N. R. S., 92, Bellevue
}

( Reçu le 19 septembre 1969)

\begin{abstract}
Résumé. - On présente ici un dispositif simple d'initiation des étincelles sous vide qui permet un amorçage précis et reproductible en vue de l'application à la spectroscopie d'absorption dans l'ultraviolet lointain. L'amorçage est commandé par une étincelle glissante auxiliaire dont le courant est une onde rectangulaire produite par la décharge de la capacité propre d'un câble coaxial dans son impédance caractéristique.

On étudie les variations du délai d'amorçage et le degré de reproductibilité en fonction d'une part de l'intensité du courant et de la durée de l'étincelle d'amorçage et, d'autre part, de la tension de l'étincelle principale et de la distance inter-électrodes. Les résultats montrent que l'on obtient une reproductibilité satisfaisante de l'instant d'amorçage $( \pm 0,1 \mu \mathrm{s})$, avec des impulsions qui mettent en jeu des énergies électriques relativement faibles, de l'ordre de quelques dizaines de millijoules.

Abstract. - A simple triggering device for vacuum sparks is presented. It allows an accurate and reproducible triggering for application to far ultraviolet absorption spectroscopy. Triggering is produced by an auxiliary sliding spark whose current is a rectangular pulse arising from the discharge of the self capacitance of a coaxial cable into the surge impedance.

The variations in the breakdown delay and the degree of reproducibility have been studied with respect both : to the current and the duration of the auxiliary spark and also to the principal spark voltage and the interelectrode gap. The results show that a good reproducibility of the breakdown delay $( \pm 0,1 \mu \mathrm{s})$ can be obtained with pulses of low electric energy i. e., of the order of a few tens of millijoules.
\end{abstract}

I. Introduction. - Les applications spectroscopiques et analytiques de l'étincelle sous vide sont particulièrement importantes dans l'ultraviolet lointain [1] [2] [3] [4] ainsi que dans le domaine des rayons $X$ [5] [6] et de plus en plus, elles font appel aux techniques de détection photoélectrique qui permettent une résolution dans le temps du spectre émis, ainsi qu'une mesure plus précise de l'intensité des raies [4] [7]. Dans tous les exemples qui viennent d'être cités, on utilise un dispositif d'amorçage qui permet de produire des étincelles dans des conditions techniquement plus aisées que celles exigées pour la décharge disruptive simple, en raison de la valeur relativement faible du champ électrique nécessaire ici (quelques $\mathrm{kV} / \mathrm{cm}$ ). En outre, un tel dispositif permet de commander le rythme de déclenchement des étincelles.

Cependant, le système d'initiation utilisé jusqu'ici est encore d'une précision et d'une reproductibilité insuffisantes pour l'analyse des phénomènes d'émission en fonction du temps au début de la décharge. Une meilleure maitrise de l'instant d'amorçage s'est révélée en outre indispensable pour les études de plasmas transitoires par spectroscopie d'absorption dans l'ultra- violet lointain [8], en vue d'assurer une synchronisation correcte du phénomène étudié et de la source (étincelle) fournissant le spectre continu.

Nous avons donc été amenés à étudier un nouveau dispositif d'initiation qui permet un réglage plus précis de l'amorçage. Signalons que ce perfectionnement technique a également rendu possible l'observation des faibles courants dans la phase de prédécharge [9].

II. Dispositif expérimental. - II.1 Circuit DE LA DÉCHARGE PRINCIPALE. - La chambre à étincelles est constituée par une enceinte métallique où la pression du gaz résiduel est abaissée à des valeurs comprises entre 2 et $5 \times 10^{-6}$ torr à l'aide d'une chaîne de pompage classique. L’étincelle est produite entre une cathode massive en acier, reliée électriquement à la masse de la chambre et une anode constituée par un cylindre d'acier de $3 \mathrm{~mm}$ de diamètre et d'une longueur supérieure à $30 \mathrm{~mm}$ (Fig. 1). La cathode est fixée sur un porte-électrode coulissant afin de pouvoir faire varier la distance inter-électrodes $d_{p}$, tout en maintenant le vide dans l'enceinte.

Le circuit de décharge de l'étincelle principale est 


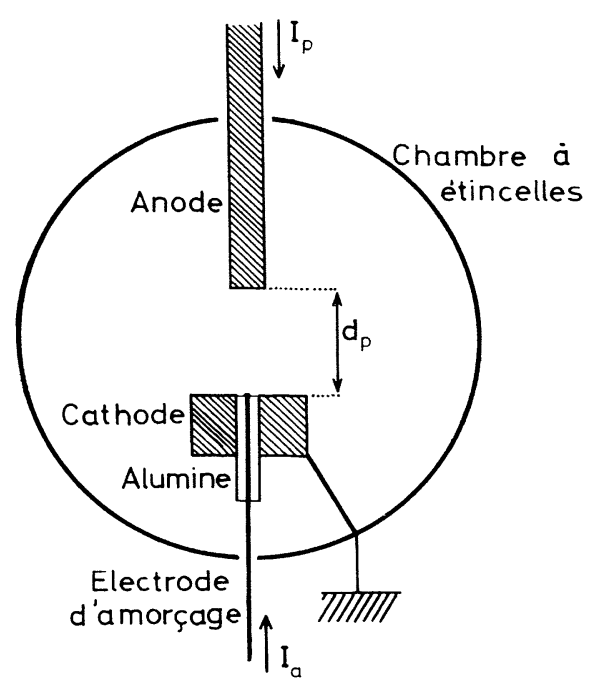

FIG. 1. - Disposition des électrodes dans la chambre à étincelles.

de type coaxial; le condensateur utilisé possède un blindage métallique relié à l'une de ses armatures. Sa capacité est de $0,3 \mu \mathrm{F}$ et il est chargé à des tensions comprises entre quelques kilovolts et $15 \mathrm{kV}$.

Un shunt également coaxial et qui sera décrit plus loin, est incorporé dans le circuit de retour du courant de décharge. Il permet l'observation du courant de l'étincelle sur l'une des voies d'un oscillographe à double faisceau.

II.2 Dispositif D'AMORÇAGE. - La décharge principale est amorcée par une étincelle glissante selon la méthode décrite par ailleurs [3]. Ce dispositif est constitué par une électrode auxiliaire isolée de la cathode par un tube d'alumine de diamètre $2 \mathrm{~mm}$ (Fig. 1). L'étincelle d'amorçage se développe donc sur la section du tube d'alumine et projette en direction de l'anode des particules chargées qui sont à l'origine d'un processus de multiplication conduisant à l'étincelle principale.

Pour créer l'étincelle d'amorçage, on a fait appel dans les travaux antérieurs à la décharge d'un condensateur, qui peut être commandée, soit par un dispositif électromécanique [3], soit par un dispositif électronique (thyratron à hydrogène) [10] [11]. Dans ce dernier cas, on adjoint un transformateur d'impulsions pour augmenter la tension aux bornes de l'initiateur et atteindre la valeur qui permet le claquage à la surface de l'alumine.

Cependant dans les dispositifs cités, le courant dans l'étincelle d'amorçage se présente sous la forme de trains oscillants de fréquence élevée, de reproductibilité médiocre et conduisant à un délai d'amorçage mal défini (Fig. 2a). Aussi ces techniques se sont montrées mal adaptées, en particulier pour l'étude que nous avons entreprise [8] des spectres d'absorption des étincelles où l'on utilise comme source continue une seconde étincelle plus brève qui doit être déclenchée à divers stades de la décharge étudiée.
De meilleurs résultats peuvent être obtenus à l'aide $\mathrm{du}$ dispositif d'amorçage que nous présentons cidessous et qui délivre une impulsion rectangulaire, unique, brève et reproductible (Fig. $2 c$ ); il utilise la
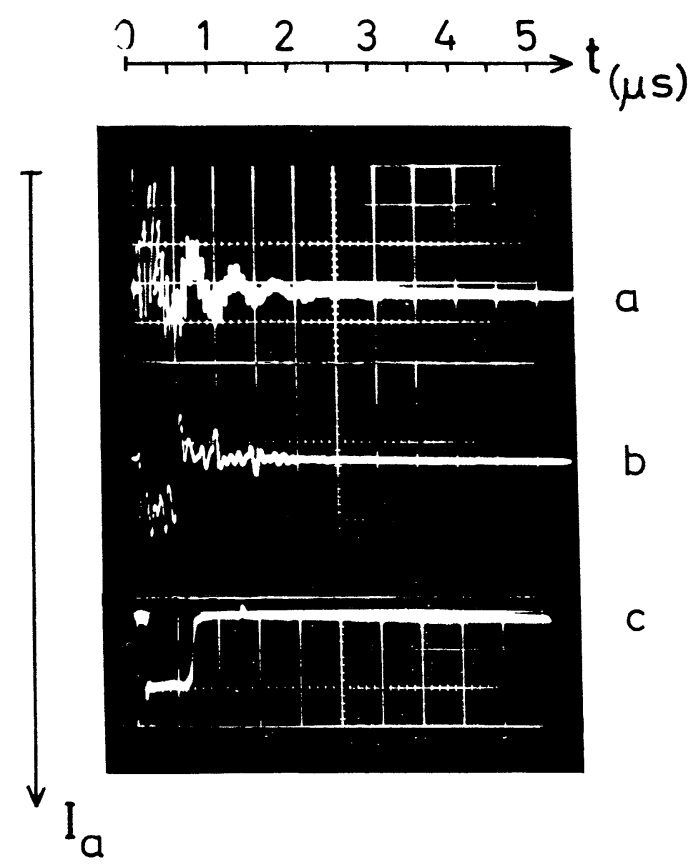

FIG. 2. - Oscillogrammes du courant dans le circuit d'amorçage a) décharge d'un condensateur,

$b$ ) et $c$ ) décharge d'un câble (en $b$, la déformation du signal est due à la self-induction du shunt de mesure).

décharge de la capacité propre d'un câble coaxial fermé sur son impédance caractéristique (Fig. 3). Le câble est chargé sous des tensions comprises entre 1,5

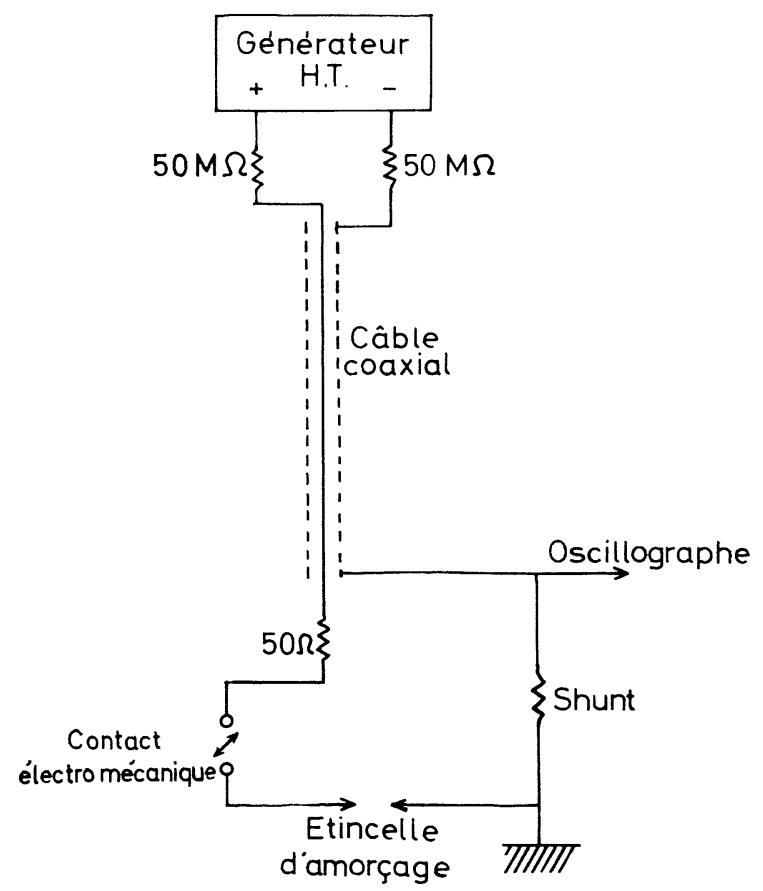

FIG. 3. - Schéma électrique du dispositif d'amorçage. 
et $12 \mathrm{kV}$ à travers une résistance de valeur élevée (100 M $\Omega$ ). La durée de l'impulsion est proportionnelle à la longueur du câble ; nous avons ainsi réalisé des impulsions de $0,1-0,5$ et $1 \mu$ s en choisissant des câbles de longueurs respectives 10,50 et 100 mètres et dont l'impédance caractéristique est $Z_{\mathrm{c}}=50 \Omega$.

Le déclenchement de l'étincelle d'amorçage est piloté par un contact mû par un système électromécanique et placé le plus près possible de la chambre. Il est inclus dans le circuit coaxial ainsi que la résistance d'adaptation du câble. Comme le courant principal, le courant de l'étincelle d'amorçage est observé par l'intermédiaire d'un shunt qui sera décrit dans le paragraphe suivant.

La valeur du courant, constante pendant toute la durée de l'impulsion, peut être déduite théoriquement de la valeur $V_{\mathrm{a}}$ de la tension à laquelle est chargé le câble : $I_{\mathrm{a}}=V_{\mathrm{a}} / 2 Z_{\mathrm{c}}$. Cette remarque est importante, car elle permet l'étalonnage des shunts de mesure.

II. 3 ShunTS DE MESURE. - On peut faire appel à deux méthodes pour enregistrer l'évolution du courant de décharge dans les étincelles sous vide : la ceinture de Rogowski et le shunt résistant.

La première solution présente des avantages pratiques : simplicité, indépendance électrique du circuit de décharge ; cependant elle n'a pas été retenue pour cette étude en régime non sinusoïdal, car son gain étant fonction de la fréquence, les variations rapides de courant sont plus amplifiées et l'on observe une image déformée du courant réel.

La deuxième solution serait excellente si l'on pouvait disposer d'un shunt absolument exempt de selfinduction et le problème pratique consiste à réaliser de tels shunts. En effet, les difficultés apparues lors de la réalisation des shunts de mesure sont liées à la présence d'harmoniques de fréquence élevée (plusieurs $\mathrm{MHz}$ ) dans les courbes de courant : l'avance de phase $L \omega / R$ introduite par le shunt devient rapidement importante et le signal observé se rapproche de la dérivée du courant. D'autre part, l'ordre de grandeur des courants maximaux mesurés $\left(10^{2} \mathrm{~A}\right.$ pour le courant d'amorçage et $10^{4} \mathrm{~A}$ pour le courant principal) impose le choix de valeurs faibles pour $R$ (de l'ordre de $0,1 \Omega$ ) ce qui augmente encore le rapport $L / R$. Nous nous sommes donc attachés à rechercher pour le shunt des formes géométriques et des matériaux tels que le rapport $L / R$ soit aussi faible que possible. Dans une première réalisation, le shunt était constitué d'un fil d'alliage résistant (Nickel-Chrome) de $100 \mathrm{~mm}$ de longueur et de $0,5 \mathrm{~mm}$ de diamètre ; la figure $2 b$ montre le signal observé dans ces conditions pour le courant d'amorçage. En fait, la dérivation du front de montée de l'impulsion montre que la quantité $L / R$ est encore trop élevée. D'autres montages analogues ont été tentés avec divers matériaux sans améliorations notables, aussi avons-nous été amenés à considérer qu'en raison de la difficulté pour diminuer le rapport $L / R$ pour les faibles valeurs de $R$, il était préférable de placer en parallèle plusieurs éléments de résistance plus élevée mais possédant chacun, de ce fait, un rapport $L / R$ plus favorable. On a alors sensiblement la même valeur du rapport pour l'ensemble du shunt. Dans ces conditions (*), on obtient un signal rectangulaire tout à fait acceptable, représenté par l'oscillogramme c de la figure 2 .

$\mathrm{Au}$ point de vue pratique, le shunt de mesure du courant principal comporte 25 résistances de $4,7 \Omega$ disposées sur les génératrices d'un cylindre de $100 \mathrm{~mm}$ de diamètre. Il est placé entre la cathode et l'armature négative du condensateur. Pour l'observation du courant d'amorçage, on utilise un shunt construit sur le même modèle mais qui ne comporte que 10 résistances, le courant qui les traverse étant plus faible.

Les deux shunts ont été étalonnés aux fréquences d'utilisation à l'aide d'impulsions analogues à la décharge utilisée pour l'amorçage. On déduit la résistance effective des shunts en mesurant la tension aux bornes et en calculant le courant par la relation $I_{\mathrm{a}}=V_{\mathrm{a}} / 2 Z_{\mathrm{c}}$ où $V_{\mathrm{a}}$ représente la tension de charge de câble, connue par ailleurs. On obtient $0,55 \Omega$ pour le shunt du circuit d'amorçage et $0,2 \Omega$ pour le shunt du circuit principal.

III. Etude du délai d'amorçage. - La qualité principale que l'on attend d'un dispositif d'initiation concerne le degré de reproductibilité de l'amorçage et en particulier du délai qui s'écoule entre l'impulsion électrique d'initiation et la décharge principale. Les caractéristiques et les propriétés d'un dispositif donné, tel que celui décrit ci-dessus, dépendent de plusieurs facteurs ; nous avons étudié d'une part l'influence des différents paramètres de l'étincelle auxiliaire et de la décharge principale sur le délai d'amorçage et d'autre part le degré de reproductibilité de ce délai dans les divers cas. Cette étude a été effectuée dans un domaine assez étendu afin de permettre le choix des conditions d'amorçage les plus avantageuses correspondant à chaque application particulière ; en effet les conditions pratiques imposées pour ces applications (tension et distance inter-électrodes) ne sont pas toujours les plus favorables pour l'amorçage (par exemple tension relativement faible pour certaines applications analytiques).

Les grandeurs qui interviendront dans les séries de mesures effectuées sont les suivantes :

$I_{\mathrm{a}}$ : intensité du courant dans l'étincelle d'amorçage ; $\tau_{\mathrm{a}}$ : durée de l'étincelle d'amorçage ;

$d_{\mathrm{p}}$ : distance entre l'anode et la cathode de la décharge principale ;

$V_{\mathrm{p}}$ : tension initiale de charge du condensateur principal ;

(*) Des essais ont été effectués avec des résistances non inductives à couche métallique; mais celles-ci ont été rapidement détériorées. Il a donc été nécessaire de faire appel à des résistances bobinées vitrifiées, dont les sens de bobinage ont été inversés d'une résistance à l'autre. 
$I_{\mathrm{p}}(t)$ : intensité du courant dans l'étincelle principale ;

$\Delta t$ : retard à l'amorçage; ce délai représente le temps qui sépare le front de montée de l'impulsion d'amorçage et l'instant où se produit l'avalanche de la décharge principale repérée par la variation brusque de la dérivée $\mathrm{d} I_{\mathrm{p}} / \mathrm{d} t \mathrm{du}$ courant principal (coude observé sur les oscillogrammes du courant, à mieux que $0,1 \mu$ s près).

On a tout d'abord étudié les variations de $\Delta t$ en fonction du courant de l'impulsion d'amorçage, ceci pour différentes valeurs de $V_{\mathrm{p}}$. Les résultats sont donnés sur la figure 4 ; les points expérimentaux représentent la valeur moyenne de $\Delta t$ prise sur dix étincelles successives. Les barres verticales correspondent aux écarts maximaux observés sur les oscillogrammes.

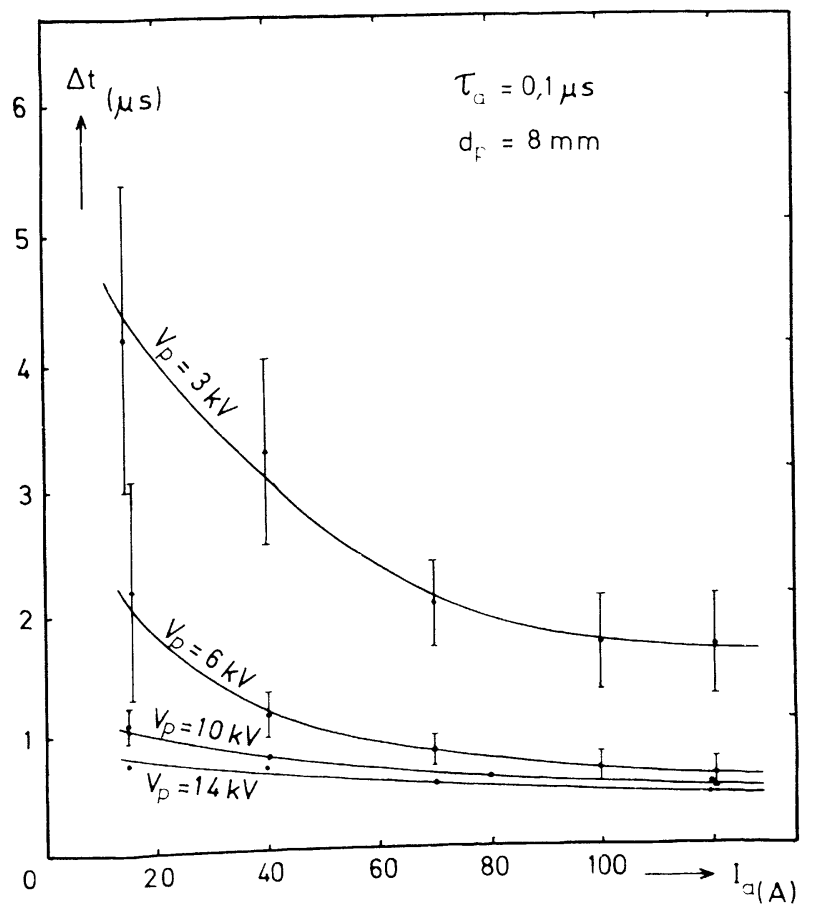

Fig. 4. - Variation du délai d'amorçage en fonction du courant dans l'étincelle d'amorçage pour plusieurs valeurs de la tension principale $V_{\mathrm{p}}$ (les barres verticales représentent l'écart maximal observé sur une dizaine d'étincelles).

On a examiné ensuite l'influence sur $\Delta t$ de la durée de l'impulsion d'amorçage et celle de la distance inter-électrodes $d_{\mathrm{p}}$. Les résultats obtenus sont représentés sur la figure 5 par les courbes $\Delta t\left(d_{\mathrm{p}}\right)$ pour $\tau_{\mathrm{a}}=0,1 \mu \mathrm{s}$ et $\tau_{\mathrm{a}}=0,5 \mu \mathrm{s}$. A titre d'illustration, on présente une série d'oscillogrammes (Fig. 6) qui montrent l'évolution du courant $I_{\mathrm{p}}(t)$ dans la décharge principale pour six valeurs croissantes de la distance $d_{\mathrm{p}}$. Dans chacun des cas, on a superposé les traces correspondant à dix étincelles successives.

Enfin la figure 7 montre les variations du délai d'amorçage en fonction de la tension principale $V_{\mathrm{p}}$

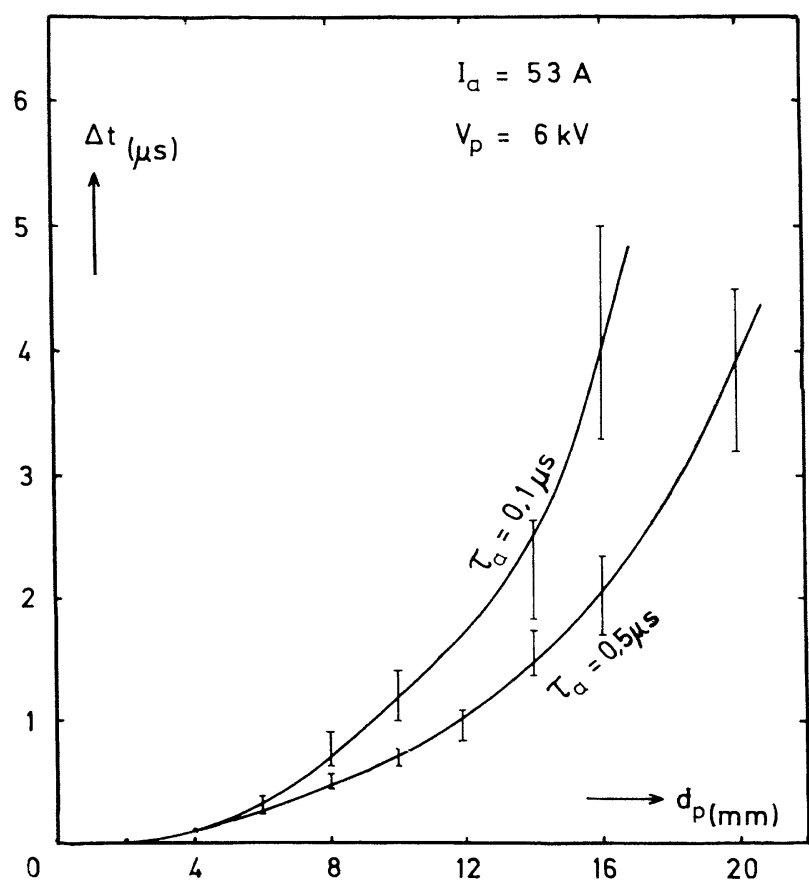

Fig. 5. - Variation du délai d'amorçage en fonction de la distance inter-électrodes pour deux valeurs $\tau_{\mathrm{a}}=0,1 \mu \mathrm{s}$ et $\tau_{\mathrm{a}}=0,5 \mu \mathrm{s}$ de la durée de l'impulsion d'amorçage.

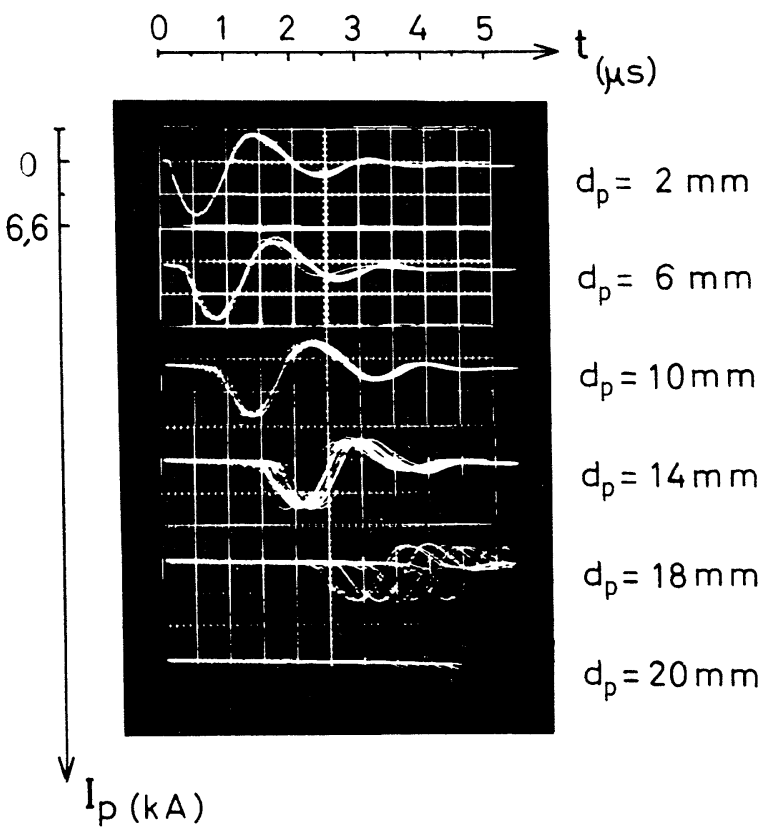

FIg. 6. - Oscillogrammes du courant dans l'étincelle principale pour diverses valeurs de la distance inter-électrodes. (Pour chaque valeur de $d_{\mathrm{p}}$ on a superposé dix traces correspondant à dix étincelles successives.)

pour trois valeurs de $d_{\mathrm{p}}$. Ces courbes représentent la valeur moyenne de $\Delta t$ effectuée cette fois sur une cinquantaine d'étincelles.

L'ensemble de ces données expérimentales indique que le délai d'amorçage dépend très fortement de la distance inter-électrodes. Les figures 5 et 7 montrent 


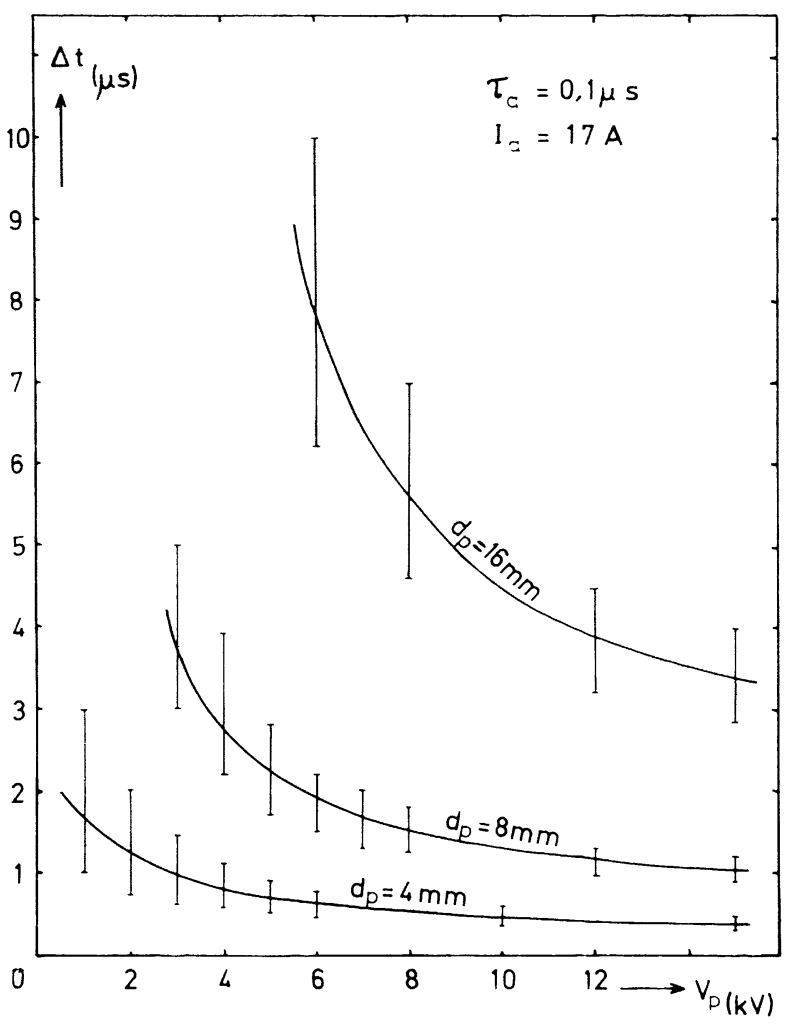

FIG. 7. - Variation du délai d'amorçage en fonction de la tension principale pour plusieurs valeurs de la distance interélectrodes.

que $\Delta t$ est une fonction sensiblement exponentielle de $d_{\mathrm{p}}$.

On constate que la variation de $\Delta t$ avec la tension $V_{\mathrm{p}}$ est beaucoup plus faible dans la gamme des tensions élevées qui représente le domaine habituel des étincelles sous vide. Au contraire, pour les tensions très faibles, l'amorçage est critique et ne peut être obtenu de façon reproductible qu'en faisant appel à des impulsions d'amorçage de longue durée.

A ces deux domaines de tension correspondent, semble-t-il, deux régimes différents de la décharge, qui sont mis en évidence par les courbes de la figure 8 . Sur cette figure, on donne l'évolution en coordonnées logarithmiques du courant $I_{\mathrm{p}}$ de la décharge principale tout au long de la phase de formation de l'étincelle pour deux valeurs de la tension $V_{\mathrm{p}}$. Ces courbes sont obtenues en enregistrant la montée du courant (en prenant comme précédemment les valeurs moyennes sur dix étincelles) avec des sensibilités de plus en plus grandes pour observer la phase initiale des phénomènes.

La courbe $V_{\mathrm{p}}=760 \mathrm{~V}$ présente une montée pratiquement exponentielle entre 0,1 et $0,7 \mu$ s puis une croissance plus lente traduisant la baisse de tension aux bornes des électrodes. On obtient dans ces conditions une décharge non oscillante.

Dans le cas d'une tension $V_{\mathrm{p}}$ plus élevée (courbe $V_{\mathrm{p}}=4000 \mathrm{~V}$ ), on trouve également une pente constante en coordonnées logarithmiques de 0,1 à $0,6 \mu \mathrm{s}$, mais on assiste ensuite à une croissance beaucoup

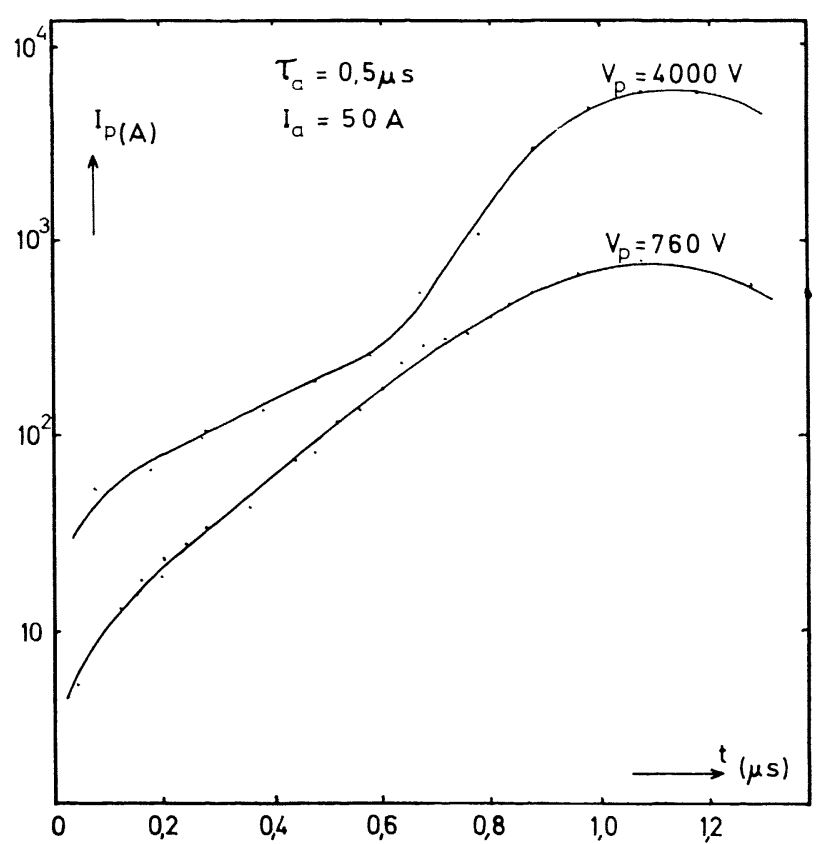

FIG. 8. - Evolution du courant dans l'étincelle principale pendant les premiers instants de la décharge pour deux régimes d'étincelle correspondant à des valeurs très différentes de la tension principale (l'instant initial correspond au front de montée de l'impulsion d'amorçage).

plus rapide du courant; cette phase correspond à la décharge proprement dite qui est celle que l'on observe sur les oscillogrammes de la figure 6.

Il semble que le régime de prédécharge puisse s'interpréter par un phénomène de multiplication au niveau des électrodes : les électrons du plasma de l'étincelle auxiliaire sont accélérés dans le champ principal et viennent bombarder l'anode provoquant ainsi une émission de particules chargées qui, à leur tour, produisent par impact sur la cathode, des électrons secondaires. Ceci engage un processus de multiplication qui correspond bien à une évolution exponentielle du courant $I_{\mathrm{p}}$.

La croissance brusque observée sur la courbe $V_{\mathrm{p}}=4000 \mathrm{~V}$ à partir de $0,7 \mu$ s s'expliquerait par la superposition d'un nouveau phénomène d'avalanche déclenché par l'apparition de vapeurs anodiques qui ont déjà été mises en évidence dans des conditions analogues [12] [13]. Ces vapeurs sont ionisées par chocs électroniques, d'où production d'électrons secondaires à la cathode par bombardement par les ions formés. Ce mécanisme divergent conduit à l’arc.

L'examen de la dispersion des résultats (barres verticales) sur les diverses courbes montre que la reproductibilité dans l'amorçage dépend essentiellement de la valeur du délai $\Delta t$. On constate que pour des retards inférieurs à une microseconde la reproductibilité reste dans des limites satisfaisantes de l'ordre de $\pm 0,2 \mu \mathrm{s}$. Cependant la durée $\tau_{\mathrm{a}}$ de l'impulsion d'amorçage joue également un rôle, illustré sur la figure 5 : pour un même délai la dispersion est moins importante sur la courbe $\tau_{\mathrm{a}}=0,5 \mu \mathrm{s}$. 
Il est possible ainsi de déterminer le domaine d'utilisation du système d'amorçage; on constate sur la figure 4 que pour les valeurs usuelles de $V_{\mathrm{p}}$ et $d_{\mathrm{p}}$ $(10 \mathrm{kV}, 8 \mathrm{~mm})$ ces conditions sont déjà réalisées avec des impulsions d'amorçage relativement faibles $\left(I_{\mathrm{a}}=20 \mathrm{~A}, \tau_{\mathrm{a}}=0,1 \mu \mathrm{s}\right)$ qui mettent en jeu des énergies électriques de l'ordre de $2 \times 10^{-3} \mathrm{~J}$. Cette valeur est environ mille fois inférieure à celle de l'énergie qui est emmagasinée dans le condensateur utilisé dans les premiers dispositifs d'amorçage [3] et elle est encore cent fois plus faible que celle qui correspond aux systèmes de déclenchement électroniques [10].

Signalons enfin que dans le cas où le champélectrique principal est plus faible, on peut encore obtenir une bonne reproductibilité en choisissant un câble plus long et chargé à des tensions élevées.

Conclusion. - Les résultats décrits ci-dessus montrent clairement l'avantage d'une impulsion d'amorçage rectangulaire par rapport à une onde oscillante. Le choix des caractéristiques optimales du circuit d'amorçage dépend du retard à l'amorçage admis ou désiré. D’une façon générale les résultats décrits cidessus font apparaître l'intérêt, au point de vue de la reproductibilité, de choisir une impulsion aussi longue que possible mais qu'il est évidemment inutile de prolonger au-delà de l'amorçage.

En vue des applications à la spectroscopie d'absorption des plasmas d'étincelles, utilisant comme source continue une autre étincelle plus brève, le facteur essentiel est la reproductibilité. Cette condition impose une limite supérieure au retard à l'amorçage admissible comme il a été indiqué plus haut. Si l'on exige ici une erreur inférieure à $\pm 0,1 \mu$ s, même dans le cas le plus favorable $\left(\tau_{\mathrm{a}}=0,5 \mu \mathrm{s}\right)$ le retard ne doit pas dépasser $0,7 \mu$ s comme le montre la figure 5 (pour $d_{\mathrm{p}}=10 \mathrm{~mm}$ ). Dans les expériences où l'on souhaite un décalage entre les deux étincelles supérieur à cette valeur, il conviendra d'introduire ce délai par un dispositif électronique annexe au niveau des circuits commandant les deux impulsions d'amorçage.

\section{Bibliographie}

[1] SAmson (J. A. R.), Techniques of Vacuum Ultraviolet Spectroscopy, John Wiley and Sons, New York, $1967,163$.

[2] Zaidel (A. N.), Schreider (E. J.), Spectroscopia Vacuumnogo Ultravioleta Physica i Technica Spectralnovo Analysa, Moscou, 1967.

[3] Balloffet (G.), Ann. Phys., Paris, 1960, 5, 1243.

[4] Berneron (R.) et Romand (J.), Mem. Sci. Rev. Metall., 1964, 61, 20.

[5] Flynn (P. T. G.), Proc. Phys. Soc. (London), 1956, $69 \mathrm{~B}, 748$.

[6] Handel (S. K.), Arkiv for Fysik, 1964, 28, 303.

[7] Romand (J.) et Berneron (R.), XIII Colloqiuum Spectroscopicum Internationale (Ottawa. 1967), Adam Hilger Ltd (London), 434.
[8] Mehlman-Balloffet (G.) et Esteva (J. M.), Astro phys. Journal, 1969, 157, 945.

[9] Esteva (J. M.), Bon (M.), Romand (J.), III e Symposium International, Les décharges et l'isolement électrique dans le vide, Paris, 1968, p. 373.

[10] Damany (H.), Roncin (J. Y.), Damany-Astoin (N.), Applied Optics, 1966, 5, 297.

[11] Turkey de Beauregard (H.), Morlais (M.), Robin (S.), Rev. Phys. Appl., 1968, 3, 25.

[12] Jamet (F.), C. R. Acad. Sci., 1967, 264 B, 1186.

[13] Goldman (A.), Goldman (M.), Rautureau (M.), Tchoubar (C.), J. Phys., 1965, 26, 486. 